\title{
NANOCOMPÓSITOS DE CARBONO COMO SUPORTE NA BIOENGENHARIA TECIDUAL - NANOGOBIOTEC
}

\section{Ingrid Rosales Costa ${ }^{1}$; Natália Zago Sentena ${ }^{2}$; Altevir Rossato Viana ${ }^{3}$; Cristiano Rodrigo Bohn Rhoden;; Francine Carla Cadoná5; Sergio Roberto Mortari ${ }^{6}$}

\section{RESUMO}

O óxido de grafeno (GO) é um nanomaterial que possui na sua superície grupos epóxi, hidroxilas, carbonilas e carboxilas e esses grupos promovem interações biológicas sendo possível a utilização como revestimento na engenharia tecidual. Sendo assim o presente trabalho teve como objetivo produzir nanocompositos contendo polímeros e GO como suporte, afim de avaliar o crescimento tridimensional de células, matriz extracelular e diferenciação celular. O GO foi produzido segundo Salles (2020), onde o grafite foi oxidado com $\mathrm{KMnO} 4$ em meio de ácido sulfúrico. Para os nanocompositos, duas técnicas foram utilizadas: evaporação de solvente e prensa hidráulica. Após, os mesmos foram caracterizador pela técnica de Difração de Raios X (DRX) e ensaios de viabiliadade celular in vitro de MTT. Pelos resultados obtidos, o método de evaporação de solvente não foi eficaz para a formação dos nanocompósitos. Já o método de prensagem do polimero:GO mostrou-se rápido e eficiente.

Palavras-chave: Cultura Celular; Nanomateriais de Carbono; Polímeros.

Eixo Temático: Tecnologia, Inovação e Desenvolvimento Sustentável (TIDS).

\section{INTRODUÇÃO}

O carbono é um dos elementos mais abundantes existentes na natureza e pode ser encontrado em diversas formas alotrópicas estáveis que variam do diamante, o carbono amorfo, grafite, nanotubo de carbono, fulereno e o grafeno (DRESSELHAUS, 2004).

O grafeno consiste em uma folha plana de átomos de carbono, densamente compactados e com espessura de apenas um átomo, configurados em uma estrutura cristalina hexagonal semelhante a favos-de-mel, possuindo propriedades únicas, como alta condutividade térmica e mobilidade de carga, elevada resistência mecânica, módulo de elasticidade e alta área superficial (NUNES FILHO, 2021).

Já o óxido de grafeno (GO) é um nanomaterial que possui em sua superfície grupos epóxi, hidroxilas, carbonilas e carboxilas, esses grupos promovem interações biológicas, permitindo a sua utilização como revestimento na Engenharia Tecidual, a partir disto, diversas pesquisas avaliam a incorporação de cargas de GO em polímeros e avaliam a sua influência sobre o nanocompósito resultante (Rhoden et al, 2017).

Os nanocompósitos poliméricos têm atraído grande interesse, visto que estes apresentam propriedades físico-químicas diferentes entre os micro e macrocompósitos, em comparação com os polímeros puros, os nanocompósitos poliméricos apresentam uma série de propriedades diferenciadas, tais como propriedades de

\footnotetext{
1 Ingrid Rosales Costa - Universidade Franciscana ingrid.costa@ufn.edu.br

2 Natália Zago Sentena - Universidade Franciscana natalia.zago@ufn.edu.br

3 Altevir Rossato Viana - Universidade Franciscana altevir.viana@ufn.edu.br

${ }^{4}$ Cristiano Rodrigo Bohn Rhoden - Universidade Franciscana cristianorbr@ufn.edu.br

5 Francine Carla Cadoná - Universidade Franciscana f.cadona@ufn.edu.br

${ }^{6}$ Sergio Roberto Mortari - Universidade Franciscana mortari@ufn.edu.br
} 
barreira, aumento do módulo de elasticidade e da força de tração, retardância a chama, resistência à corrosão (Rhoden et al, 2021).

Além disso os biopolímeros são bastante utilizados por sua boa biocompatibilidade e biodegradabilidade como por exemplo a quitosana que vem sendo bastante investigada por ser um biopolímero natural encontrado em exoesqueletos de crustáceos, que se dissolve facilmente em ácidos orgânicos diluídos e que tem capacidade de formar filmes, fibras ou géis (LAVORGNA, 2010).

Além dos biopolímeros também são utilizados copolímeros, como o Eudragit sendo derivado do ácido acrílico e éster metílico do ácido metacrílico. Atualmente, esse copolímero possui aplicação na área de liberação controlada de fármacos, citocompatibilidade, biodegradabilidade e estabilidade em meio aquoso (em soluções de $\mathrm{pH}<6.5)$ (PATRA, 2017)

\section{METODOLOGIA}

\section{Produção do óxido de grafeno (GO)}

O GO foi preparado conforme Salles et al (2020), um método adaptado de Hummers. Neste método o grafite é oxidado pelo tratamento com $\mathrm{KMnO}_{4}$ na presença de $\mathrm{H}_{2} \mathrm{SO}_{4}$ concentrado.

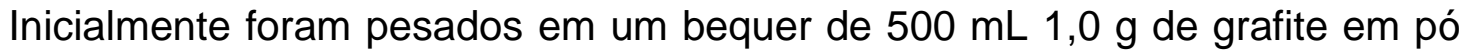
(Sigma-Aldrich, $<20 \mu \mathrm{m}$, flakes) e adicionados $23,0 \mathrm{~mL}$ de $\mathrm{H}_{2} \mathrm{SO}_{4}$ concentrado (P.A, 95-98\%, Synth) e 0,5 g de $\mathrm{NaNO}_{3}$ (P.A, Merck) (Figura $1 \mathrm{a}$ ). $\mathrm{O}$ bequer contendo a mistura mencionada acima foi colocado em banho de gelo e com agitação magnética. Lentamente foram adicionados $3,0 \mathrm{~g}$ de $\mathrm{KMnO}_{4}$ (P.A., Synth), mantendo-se a mistura sob forte agitação magnética por 60 minutos, em seguida, 46,0 mL de água destilada foram adicionados, lentamente, evitando aquecimento no meio reacional (temperatura não ultrapasse $90^{\circ} \mathrm{C}$ ).

A mistura foi mantida em agitação por 15 minutos, e após foram adicionados $10,0 \mathrm{~mL}$ de $\mathrm{H}_{2} \mathrm{O}_{2}$ (P.A., 30\% v/v, Vetec) e 140,0 mL de água deionizada (Figura $1 \mathrm{~b}$ ). O material resultante sólido (GO) foi filtrado e lavado com $250,0 \mathrm{~mL}$ de uma solução $5,0 \% \mathrm{v} / \mathrm{v}$ de $\mathrm{HCl}$ (P.A., Synth) e então lavado com água destilada inúmeras vezes, até atingir $\mathrm{pH}$ neutro (Figura $1 \mathrm{c}$ ).

Após esta etapa o mesmo foi mantido em temperatura ambiente para secagem total, para então dar início as caracterizações e produção dos nanocompósitos.

\section{Produção dos nanocompósitos}

Na produção dos nanocompósitos, foram utilizadas duas técnicas, sendo elas: a técnica de evaporação de solvente e produção com uso da prensa hidraulica.

\section{Nanocompósitos por evaporação de solvente}

Inicialmente foram utilizados os polímeros Quitosana de baixo peso molecular e Eudragit RS 100, na proporção de 1:0,05 e 2:0,05 de polimero:GO. Nesta técnica houve uma dificuldade com os nanocompósitos contendo o polímero Eudragit, pois o

\footnotetext{
${ }^{1}$ Ingrid Rosales Costa - Universidade Franciscana ingrid.costa@ufn.edu.br

2 Natália Zago Sentena - Universidade Franciscana natalia.zago@ufn.edu.br

${ }^{3}$ Altevir Rossato Viana - Universidade Franciscana altevir.viana@ufn.edu.br

${ }^{4}$ Cristiano Rodrigo Bohn Rhoden - Universidade Franciscana cristianorbr@ufn.edu.br

5 Francine Carla Cadoná - Universidade Franciscana f.cadona@ufn.edu.br

${ }^{6}$ Sergio Roberto Mortari - Universidade Franciscana mortari@ufn.edu.br
} 
filmes formados após eliminar o solvente, ficou aderido a placa de petri, sendo difícil a retirada do mesmo, obtendo-se apenas filmes de GO:Quitosana.

a)

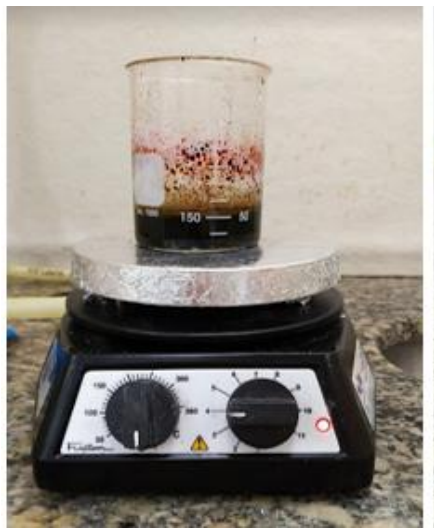

b)

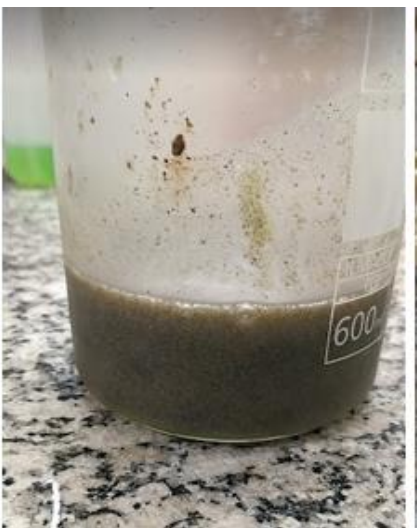

c)

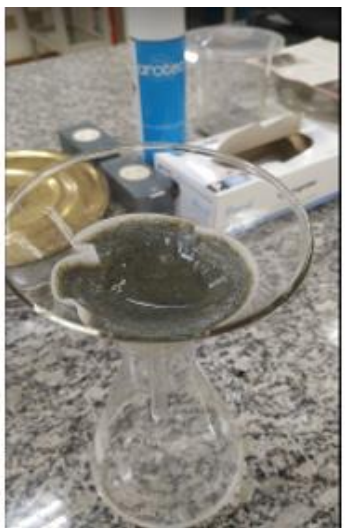

Figura 1: a) produção do óxido de grafeno (GO) b) $G O$ antes da filtragem c) Processo de filtragem do GO.

\section{Nanocompósito contendo Quitosana}

Para solubilização da quitosana foi em uma solução contendo $1 \%$ de ácido acético em água destilada. Ambos foram mantidos em agitação constante até a solubilização completa do polímero, para então unir o GO onde esta solução foi despejada em uma placa de petri até evaporação total do solvente.

\section{Nanocompósito contendo Eudragit RS 100}

Para solubilização do Eudragit foram utilizados $10 \mathrm{~mL}$ de acetona, sendo uma parte para solubilização do polímero e outra para dispersão do GO, ambos foram levados em agitação constante até solubilização total do polímero, e então feita a união com o GO, após isso a solução foi despejada em uma placa de petri para evaporação do solvente, conforme figura 2.

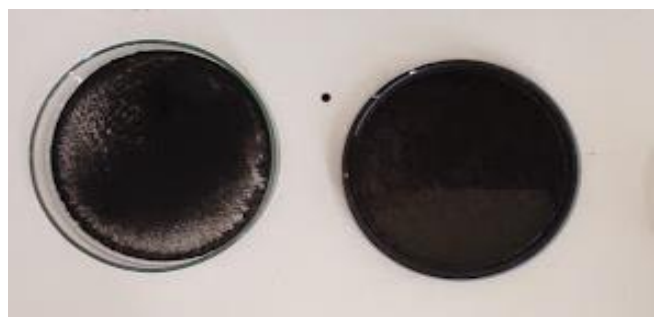

Figura 2: Nanocompósitos contendo eudragit e GO

\footnotetext{
1 Ingrid Rosales Costa - Universidade Franciscana ingrid.costa@ufn.edu.br

2 Natália Zago Sentena - Universidade Franciscana natalia.zago@ufn.edu.br

${ }^{3}$ Altevir Rossato Viana - Universidade Franciscana altevir.viana@ufn.edu.br

${ }^{4}$ Cristiano Rodrigo Bohn Rhoden - Universidade Franciscana cristianorbr@ufn.edu.br

${ }^{5}$ Francine Carla Cadoná - Universidade Franciscana f.cadona@ufn.edu.br
}

${ }^{6}$ Sergio Roberto Mortari - Universidade Franciscana mortari@ufn.edu.br 


\section{Nanocompósitos produzidos por prensa}

Nesta técnica é utilizado o equipamento chamado de prensa hidráulica manual, o qual consite em formação de um fime, conforme mostrada na figura 3. Foram utilizadas as mesmas proporções de polimero:GO (1:0,05 e 2:0,05). Nesta técnica a amostra foi prensada utilizando uma carga de 5 toneladas para a produção dos nanocompósitos, mantidos por 3 minutos.
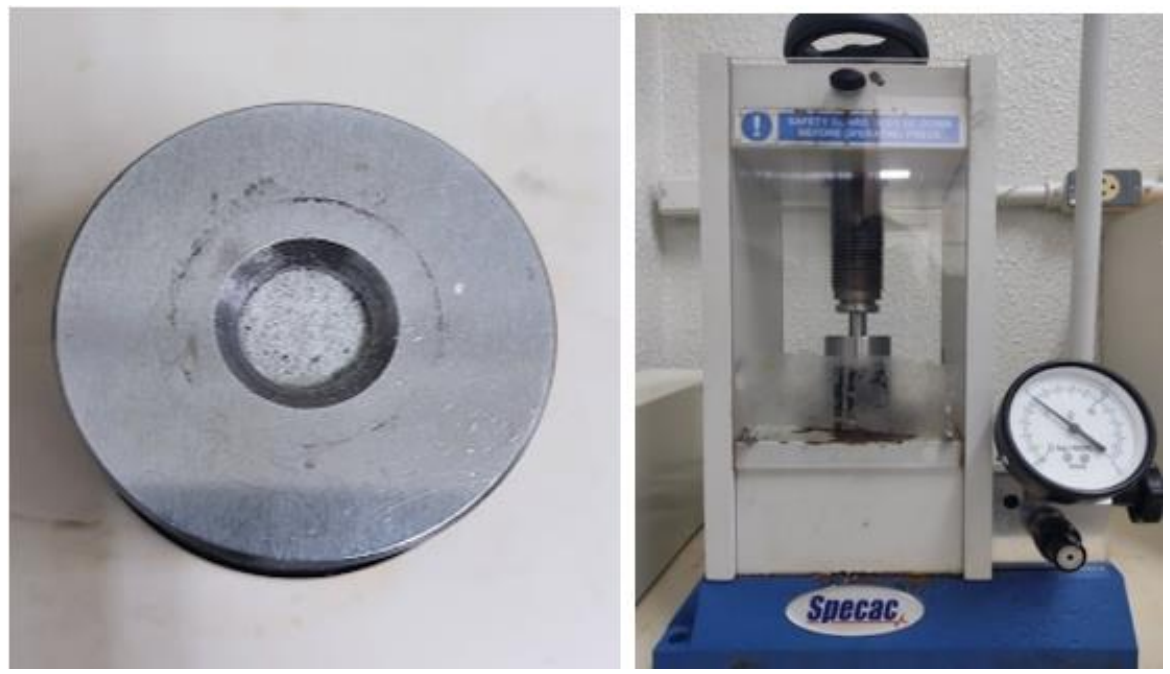

Figura 3: imagem do equipamento e porta amostra contendo polímero

Os nanocompósitos obtidos estão apresentados na figura 4.

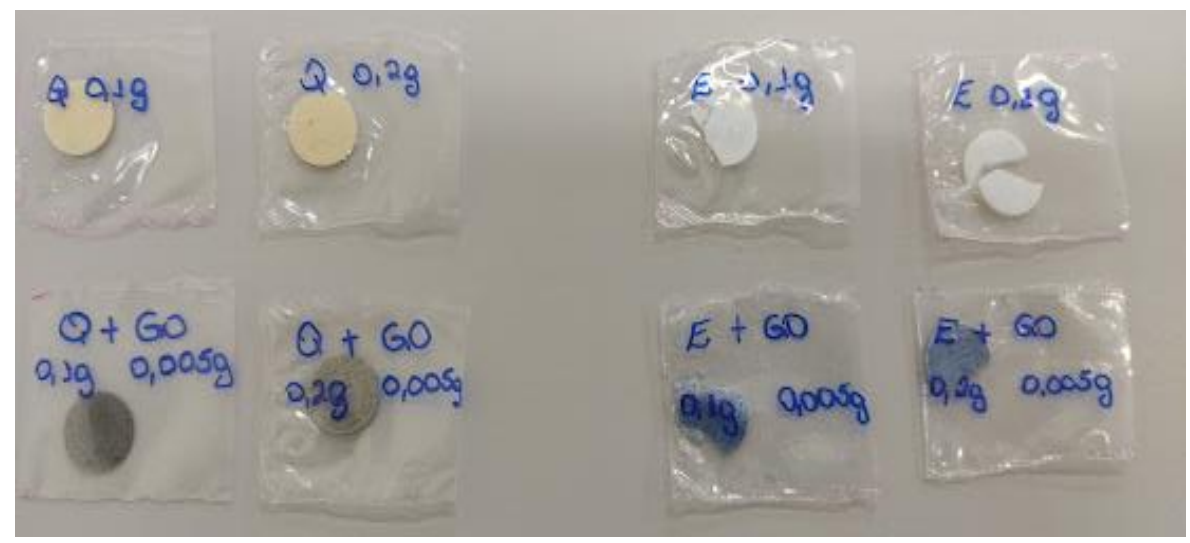

Figura 4: Nanocompósitos obtidos por meio da prensa hidráulica

\section{RESULTADOS E DISCUSSÕES}

\section{Caracterização dos nanocompósitos}

Os nanocompósitos foram caracterizados pela técnica de difração de Raio $X$ (DRX), o qual nos permite identificar os picos caracteristicos de cada material presente na amostra e também conhecer o tipo de estrutura cristalina dos materiais.

\footnotetext{
1 Ingrid Rosales Costa - Universidade Franciscana ingrid.costa@ufn.edu.br

2 Natália Zago Sentena - Universidade Franciscana natalia.zago@ufn.edu.br

${ }^{3}$ Altevir Rossato Viana - Universidade Franciscana altevir.viana@ufn.edu.br

${ }^{4}$ Cristiano Rodrigo Bohn Rhoden - Universidade Franciscana cristianorbr@ufn.edu.br

${ }^{5}$ Francine Carla Cadoná - Universidade Franciscana f.cadona@ufn.edu.br

${ }^{6}$ Sergio Roberto Mortari - Universidade Franciscana mortari@ufn.edu.br
} 
Na caracterização dos nancompósitos produzidos pela técnica de evaporação de solvente foi possível perceber que na proporção 1:0,05 (quitosana:GO) (figura $5 \mathrm{a}$ ), o difratograma não apresentou o pico caracterisco da quitosana $\left(2 \theta \approx 20^{\circ}\right)$. Já na concentração de 2:0,05 (quitosana:GO) foi possivel identificar o pico caracterisco da quitosana e do $\mathrm{GO}\left(2 \theta \approx 11^{\circ}\right)$ pelo difratograma da figura $5 \mathrm{~b}$.

a)

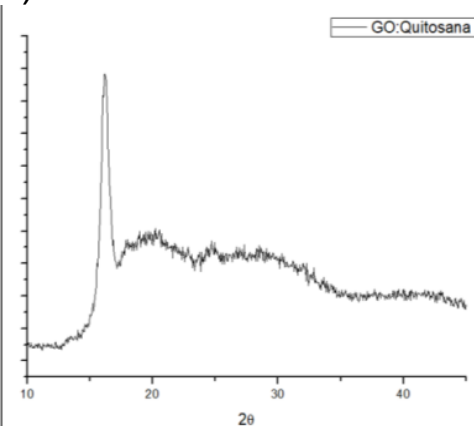

b)

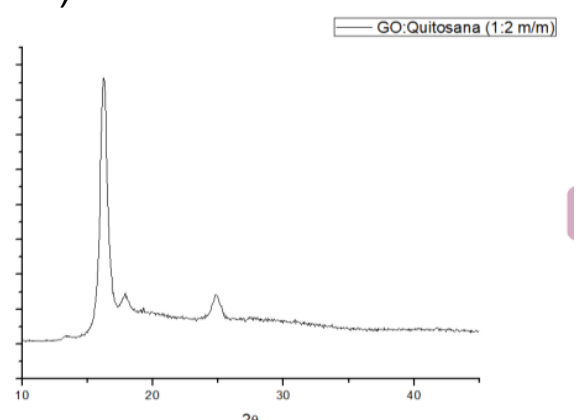

Figura 5: a) Difratograma quitosana:GO (1:0,05) b) Difratograma quitosana:GO (2:0,05).

$\mathrm{Na}$ caracterização dos nanocompósitos obtidos pela prensa hidráulica, foram obtidos resultados de 8 amostras, sendo elas brancas e contendo GO. A figura 6 apresenta o difratograma da amostra contendo $0,1 \mathrm{~g}$ (a) e $0,2 \mathrm{~g}$ (b) de quitosana ( $2 \theta \approx$ $\left.20^{\circ}\right)$, e GO:Quitosana 1:0,05 (c) e 2:0,05 (d) $\left(2 \theta \approx 11^{\circ}\right)$.

a)

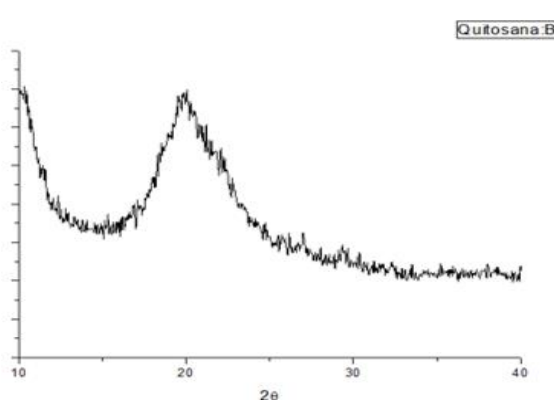

c)

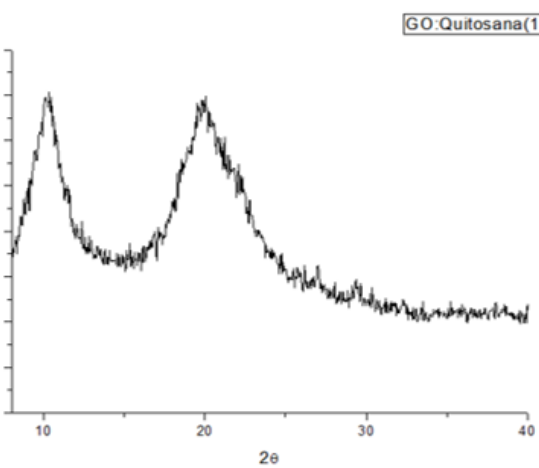

b)

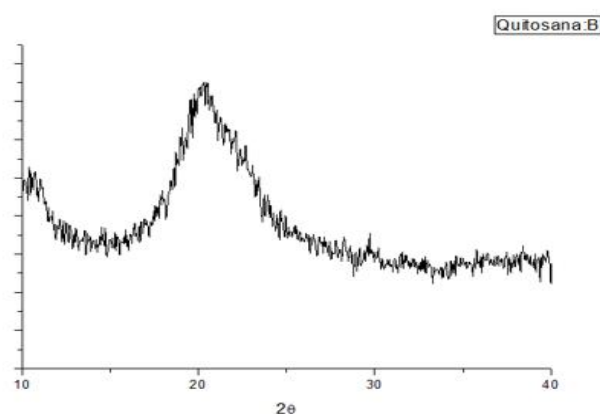

d)

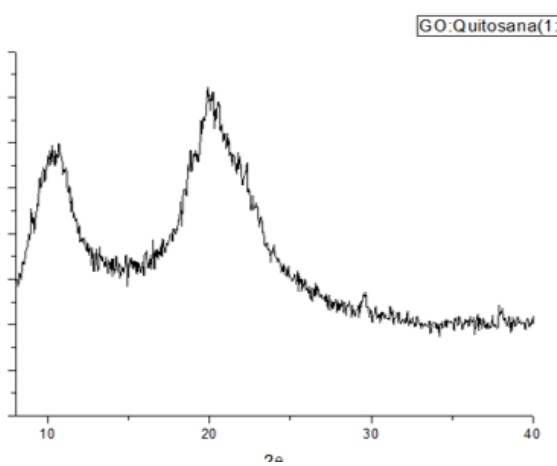

Figura 6: a) Difratograma $0,1 \mathrm{~g}$ de quitosana b) Difratograma $0,2 \mathrm{~g}$ de quitosana c) Difratograma $0,1 \mathrm{~g}$ de GO:Quitosana b) Difratograma 0,2g de GO:Quitosana.

\section{Nanocompósitos de Eudragit}

1 Ingrid Rosales Costa - Universidade Franciscana ingrid.costa@ufn.edu.br

2 Natália Zago Sentena - Universidade Franciscana natalia.zago@ufn.edu.br

${ }^{3}$ Altevir Rossato Viana - Universidade Franciscana altevir.viana@ufn.edu.br

${ }^{4}$ Cristiano Rodrigo Bohn Rhoden - Universidade Franciscana cristianorbr@ufn.edu.br

${ }^{5}$ Francine Carla Cadoná - Universidade Franciscana f.cadona@ufn.edu.br

${ }^{6}$ Sergio Roberto Mortari - Universidade Franciscana mortari@ufn.edu.br 
O difratograma da figura 7 apresenta a difração de Raio $X$ do nanocompósito Eudragit 0,1g (a) e 0,2g (b) e GO:Eudragit (c-d). Segundo a literatura Aceves (2000), a falta de picos definidos resulta em um caráter amorfo, esperado para o polímero. Nos difratogramas foi possível identificar o pico caracteristico do $\mathrm{GO}$ em $2 \theta=11^{\circ}$.

a)

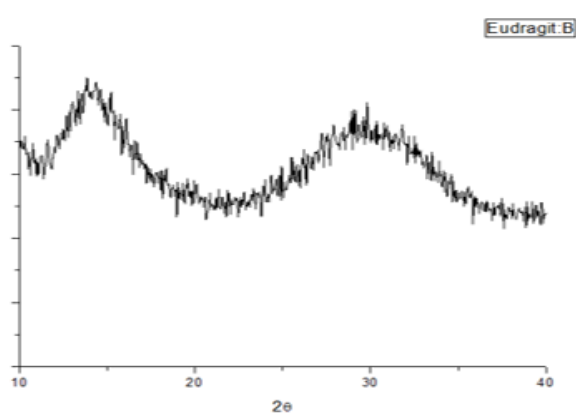

c)

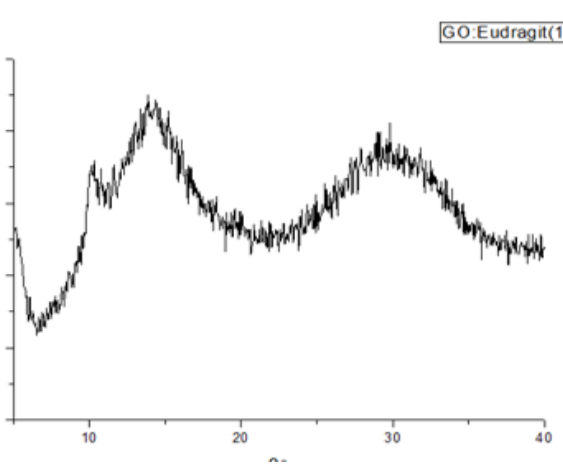

b)

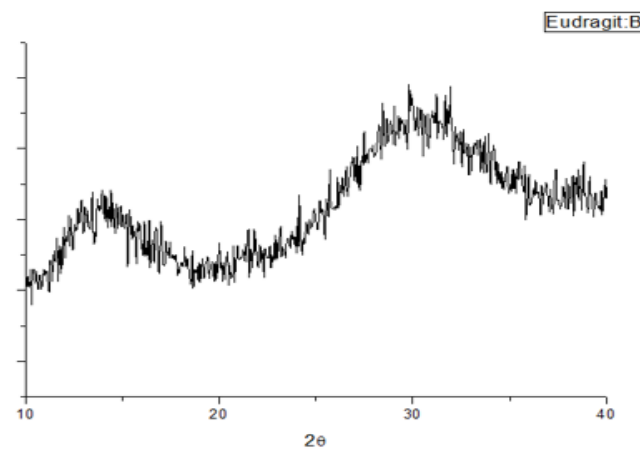

d)

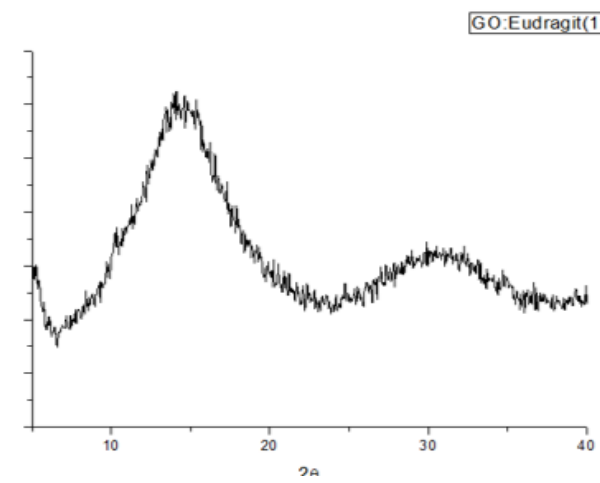

Figura 7: a) Difratograma 0,1g de Eudragit b) Difratograma 0,2g de Eudragit c) Difratograma 0,1g de GO:Eudragit b) Difratograma 0,2g de GO:Eudragit

\section{Ensaio In vitro}

Para o ensaio de MTT as amostras foram testas em células de queratinócitos humanos (figura 8). O método foi realizado de acordo com Mosmann (1983). A enzima succinate desidrogenase presente em células viáveis que metabolizam o reagente MTT é adicionado nos poços $20 \mu \mathrm{L}(5 \mathrm{mg} / \mathrm{mL}$ de PBS) onde éconvertido em MTT formazan após 4 horas de incubação. O sobrenadante é retirado e então adicionado $200 \mu \mathrm{L}$ de dimetilsulfóxido para solubilizar os cristas de cor púrpura. A leitura é feita em aparelho de ELISA no comprimento de onda de $570 \mathrm{~nm}$.

\footnotetext{
1 Ingrid Rosales Costa - Universidade Franciscana ingrid.costa@ufn.edu.br

2 Natália Zago Sentena - Universidade Franciscana natalia.zago@ufn.edu.br

${ }^{3}$ Altevir Rossato Viana - Universidade Franciscana altevir.viana@ufn.edu.br

${ }^{4}$ Cristiano Rodrigo Bohn Rhoden - Universidade Franciscana cristianorbr@ufn.edu.br

5 Francine Carla Cadoná - Universidade Franciscana f.cadona@ufn.edu.br
}

${ }^{6}$ Sergio Roberto Mortari - Universidade Franciscana mortari@ufn.edu.br 
Quanto ao teste de viabilidade celular, o nanocompósito apresentou diminuição celular na maior proporção de 0,2g de Quitosana, nas maiores concentrações (30 e 100 $\mu \mathrm{g} / \mathrm{mL}$ ), conforme figura $8(\mathrm{~b})$.

a)

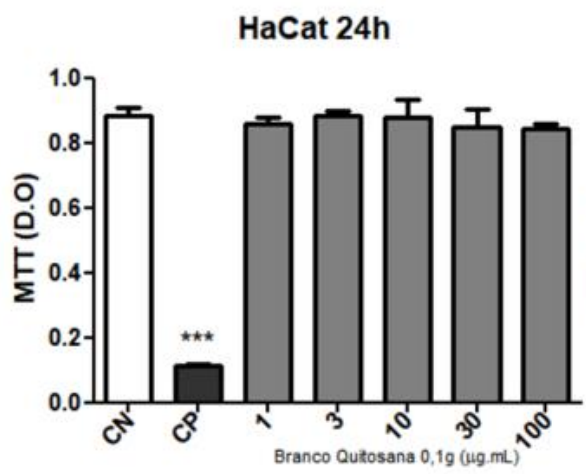

c)

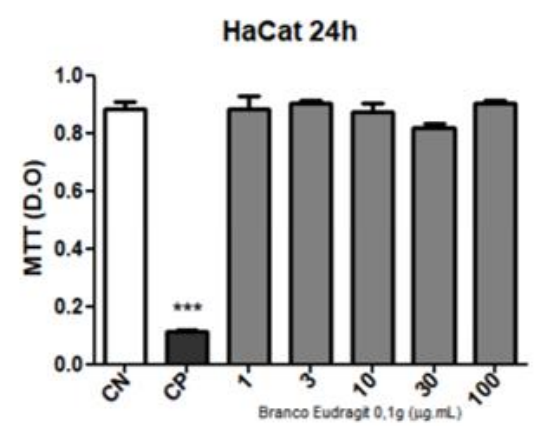

b)

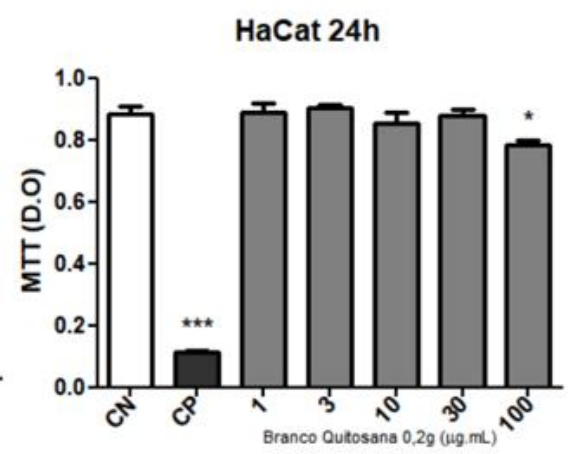

d)

HaCat 24h

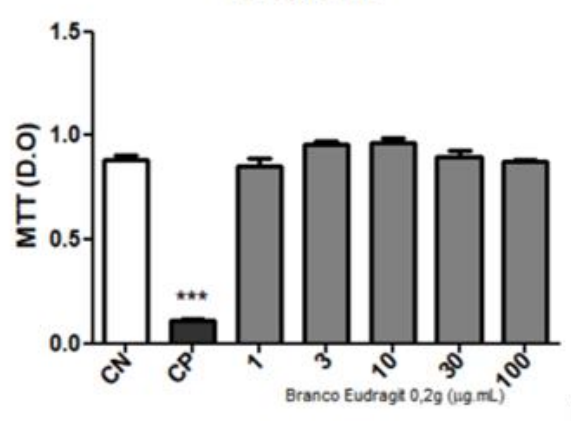

Figura 8: Teste de MTT:Eudragit 0,1 e 0,2 $\mu \mathrm{g} / \mathrm{mL}$ (a-b), Quitosana 0,1 e 0,2 $\mu \mathrm{g} / \mathrm{mL}$ (c-d)
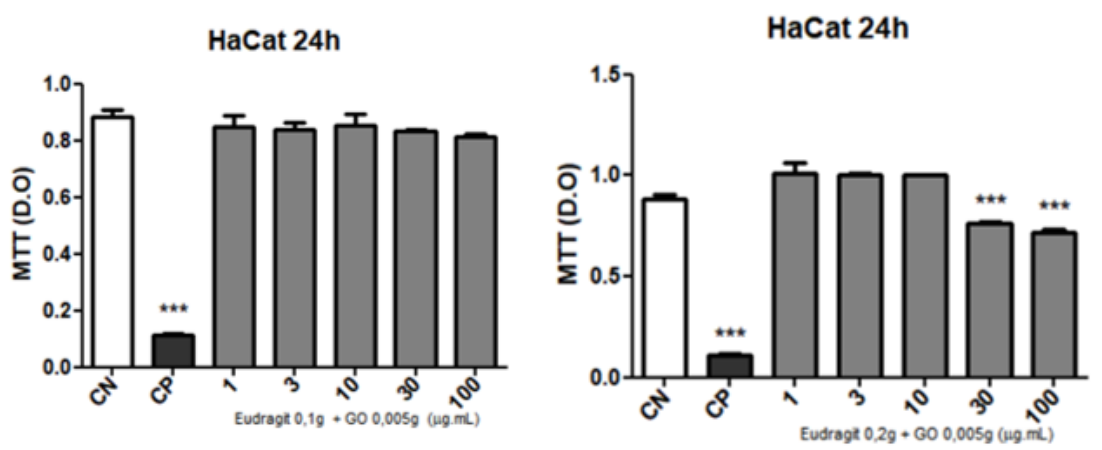

1 Ingrid Rosales Costa - Universidade Franciscana ingrid.costa@ufn.edu.br

2 Natália Zago Sentena - Universidade Franciscana natalia.zago@ufn.edu.br

${ }^{3}$ Altevir Rossato Viana - Universidade Franciscana altevir.viana@ufn.edu.br

${ }^{4}$ Cristiano Rodrigo Bohn Rhoden - Universidade Franciscana cristianorbr@ufn.edu.br

${ }^{5}$ Francine Carla Cadoná - Universidade Franciscana f.cadona@ufn.edu.br

${ }^{6}$ Sergio Roberto Mortari - Universidade Franciscana mortari@ufn.edu.br 
HaCat 24h

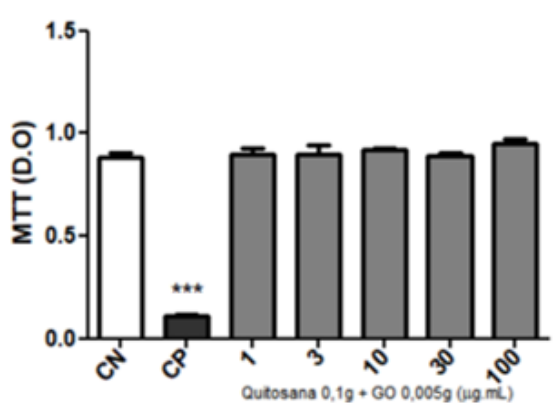

HaCat 24h

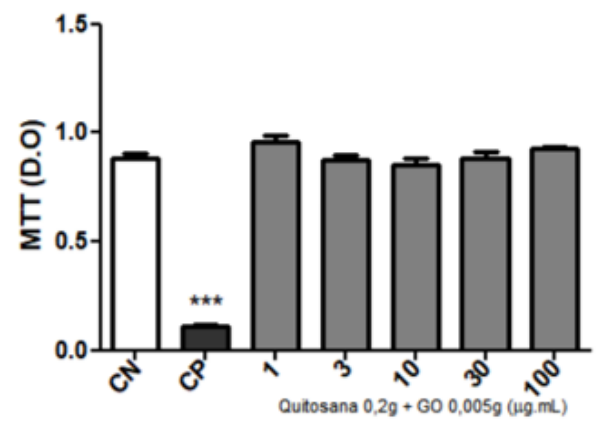

Figura 9: GO:Eudragit 1:0,05 (a) GO:Eudragit 2:0,05 (b) GO:Quitosana 1:0,05 (c) GO:Quitosana 2:0,05 (d).

Para os nanocompósitos contendo GO, apenas a amostra contendo 2:0,05 de GO:Eudragit sendo testada nas duas maiores concentrações (30 e $100 \mu \mathrm{g} / \mathrm{mL}$ ) apresentou diminuição na viabilidade celular, conforme apresenta a figura 9.

\section{CONCLUSÃO}

Conclui-se que o nanocompósito obtido por meio de prensa hidráulica proporcionou melhores resultados, pois pelo método de evaporação do solvente, o filme fica aderido ao recipiente, dificultando a sua retirada. A técnica de difração de raios $X$ mostrou 0 quanto o Eudragit é amorfo assim como a presença do GO no nanocompósito. Quanto a avaliação in vitro, os resultados mostraram que somente o GO:Eudragit $0,2 \mathrm{~g} \mathrm{e}$ quitosana 0,2g causaram citotoxicidade nas maiores concentrações utilizadas (30 e $100 \mu \mathrm{g} / \mathrm{mL}$ ). Para o nanocompósito formado por GO:Quitosana, não houve morte celular nas proporções avaliadas e tão pouco nas concentrações ate $100 \mu \mathrm{g} / \mathrm{mL}$. Ainda são necessários mais ensaios in vitro para avaliar a segurança do nanocompósito.

\section{AGRADECIMENTOS FAPERGS/UFN}

\footnotetext{
1 Ingrid Rosales Costa - Universidade Franciscana ingrid.costa@ufn.edu.br

2 Natália Zago Sentena - Universidade Franciscana natalia.zago@ufn.edu.br

${ }^{3}$ Altevir Rossato Viana - Universidade Franciscana altevir.viana@ufn.edu.br

${ }^{4}$ Cristiano Rodrigo Bohn Rhoden - Universidade Franciscana cristianorbr@ufn.edu.br

${ }^{5}$ Francine Carla Cadoná - Universidade Franciscana f.cadona@ufn.edu.br

${ }^{6}$ Sergio Roberto Mortari - Universidade Franciscana mortari@ufn.edu.br
} 


\section{REFERÊNCIAS}

ACEVES, J. M, et al. Preparation and characterization of Furosemide-Eudragit controlled release systems. International Journal Of Pharmaceutics, [S.L.], v. 195, n. 1-2, p. 45-53, fev. 2000.

DRESSELHAUS, G.; et al., UNUSUAL PROPERTIES AND STRUCTURE OF CARBON NANOTUBES. Annual Review of Materials Research, p. 34-247,2004.

FIORI, A. P. S; et al., Preparação e caracterização de nanocompósitos poliméricos baseados em quitosana e argilo minerais. Polímeros, [S.L.], v. 24, n. 5, p. 628-635, 30 set. 2014.

LAVORGNA, M.; et al,.Study of the combined effect of both clay and glycerol plasticizer on the properties of chitosan films. Carbohydrate Polymers, [S.L.], v. 82, n. 2, p. 291 298, set. 2010.

NOGUEIRA, N. M. B. Síntese de nanomateriais de grafeno para utilização em nanocompósitos poliméricos. 22f. Trabalho de conclusão de curso. Engenharia de Materiais - Universidade Federal Rural de Pernambuco, 2019.

NUNES FILHO, F. G.; et al., Híbridos de grafeno/montmorillonita e óxido de grafeno/montmorillonita como nanomateriais funcionais: uma visão da literatura atual. Cerâmica, [S.L.], v. 67, n. 382, p. 210-229, jun. 2021.

RHODEN, C. R. B.; et al. Síntese fácil e direta do óxido de grafeno magnético. Disciplinarum Scientia, Santa Maria, v. 18, n. 2, p. 389-397, ago. 2017.

RHODEN, C. R. B.; et al. Study from the influence of magnetite onto removal of hydrochlorothiazide from aqueous solutions applying magnetic graphene oxide. Journal Of Water Process Engineering, [S.L.], v. 43, p. 102-262, out. 2021.

SALLES, T.R; et al,.Graphene oxide optimization synthesis for application on laboratory of Universidade Franciscana. Disciplinarum Scientia - Ciências Naturais e Tecnológicas, [S.L.], v. 21, n. 3, p. 15-26, 2020.

\footnotetext{
1 Ingrid Rosales Costa - Universidade Franciscana ingrid.costa@ufn.edu.br

2 Natália Zago Sentena - Universidade Franciscana natalia.zago@ufn.edu.br

3 Altevir Rossato Viana - Universidade Franciscana altevir.viana@ufn.edu.br

${ }^{4}$ Cristiano Rodrigo Bohn Rhoden - Universidade Franciscana cristianorbr@ufn.edu.br

5 Francine Carla Cadoná - Universidade Franciscana f.cadona@ufn.edu.br

${ }^{6}$ Sergio Roberto Mortari - Universidade Franciscana mortari@ufn.edu.br
} 